\title{
Geometric interpretation for A-fidelity and its relation with Bures fidelity
}

\author{
Zhihao Ma, ${ }^{1} \mathrm{Fu}$-Lin Zhang, ${ }^{2}$ and Jing-Ling $\mathrm{Chen}^{2}$, * \\ ${ }^{1}$ Department of Mathematics, Shanghai Jiaotong University, Shanghai, 200240, P.R.China \\ ${ }^{2}$ Theoretical Physics Division, Chern Institute of Mathematics, Nankai University, Tianjin, 300071, P.R.China
}

(Dated: August 27, 2021)

\begin{abstract}
A geometric interpretation for the A-fidelity between two states of a qubit system is presented, which leads to an upper bound of the Bures fidelity. The metrics defined based on the A-fidelity are studied by numerical method. An alternative generalization of the A-fidelity, which has the same geometric picture, to a $N$-state quantum system is also discussed.
\end{abstract}

PACS numbers: 03.67.-a, 03.65.Ta

\section{INTRODUCTION}

The concept of fidelity plays an important role in quantum computation and quantum information [1, 2, 3, 4, [5, 6, 7]. It is a measurement of closeness between two states. The most well-known definition is the Bures fidelity between two states $\rho_{1}$ and $\rho_{2}$, given by [4, [5, 6, 7],

$$
F_{B}\left(\rho_{1}, \rho_{2}\right)=\left[\operatorname{Tr}\left(\sqrt{\sqrt{\rho_{1}} \rho_{2} \sqrt{\rho_{1}}}\right)\right]^{2} .
$$

Assume that $\rho_{1}=|\phi\rangle\langle\phi|$ and $\rho_{2}=|\varphi\rangle\langle\varphi|$ are two pure states, then $F_{B}\left(\rho_{1}, \rho_{2}\right)=|\langle\phi \mid \varphi\rangle|^{2}$. Another generalization of the usual transition probability from pure states to mixed states is the quantum affinity [8, 9]

$$
F_{A}\left(\rho_{1}, \rho_{2}\right)=\left[\operatorname{Tr}\left(\sqrt{\rho_{1}} \sqrt{\rho_{2}}\right)\right]^{2},
$$

which has many the same fundamental properties as the Bures fidelity [9]. For two pure state $\rho_{1}=|\phi\rangle\langle\phi|$ and $\rho_{2}=$ $|\varphi\rangle\langle\varphi|$, we have $F_{A}\left(\rho_{1}, \rho_{2}\right)=|\langle\phi \mid \varphi\rangle|^{4}$. Since it is denoted as $F_{A}$ in [8] by Raggio, we would like to call it A-fidelity in this report for its close relation and comparability with the Bures fidelity.

It is always interesting and useful to find an intuitive geometric picture of a concept in quantum information. For instance, the trace distance between two single qubit states has a simple geometric interpretation as half of the ordinary Euclidean distance between points on the Bloch sphere [1]. Chen and his collaborators [10] provided a geometric picture for the Bures fidelity for the qubit case. This brief report is aimed at putting forward a geometric interpretation for the A-fidelity. At first, we would like to review the result in the Bures case.

The state of a qubit is described by a $2 \times 2$ density matrix as

$$
\rho(\mathbf{n})=\frac{1}{2}(\mathbf{1}+\vec{\sigma} \cdot \mathbf{n}),|\mathbf{n}| \leq 1
$$

where 1 is the $2 \times 2$ unit matrix, $\vec{\sigma}=\left(\sigma_{1}, \sigma_{2}, \sigma_{3}\right)$ are the Pauli matrices in vector notation, and $\mathbf{n}$ is the threedimensional Bloch vector. If $|\mathbf{n}|=1$, Eq. (3) corresponds

*Email chenjl@nankai.edu.cn to a pure state, otherwise a mixed state. Chen et al. introduced the hyperbolic parameter $\phi_{\mathbf{n}}$, called rapidity, to represent the Bloch vector by the equation

$$
\mathbf{n}=\hat{\mathbf{n}} \tanh \phi_{\mathbf{n}}
$$

where $\hat{\mathbf{n}}$ is the unit vector in the direction of $\mathbf{n}$. The state in Eq. (3) is represented as

$$
\rho(\mathbf{n})=\frac{L(\mathbf{n})}{2 \cosh \phi_{\mathbf{n}}},
$$

where $L(\mathbf{n})$ is the Lorentz boost matrix,

$$
\begin{aligned}
L(\mathbf{n}) & =\exp \left(\frac{\varphi_{\mathbf{n}}}{2} \vec{\sigma} \cdot \hat{\mathbf{n}}\right) \\
& =\mathbf{1} \cosh \frac{\varphi_{\mathbf{n}}}{2}+\vec{\sigma} \cdot \hat{\mathbf{n}} \sinh \frac{\varphi_{\mathbf{n}}}{2} \\
\phi_{\mathbf{n}} & =\varphi_{\mathbf{n}} / 2 .
\end{aligned}
$$

The Bloch vector $\mathbf{n}$ corresponds to a relativistically admissible velocity, with the vacuum speed of light $c=1$. Then, the Bures fidelity between two states

$$
\begin{aligned}
& \rho_{1}=\frac{1}{2}(\mathbf{1}+\vec{\sigma} \cdot \mathbf{u}), \\
& \rho_{2}=\frac{1}{2}(\mathbf{1}+\vec{\sigma} \cdot \mathbf{v}),
\end{aligned}
$$

is given by

$$
F_{B}\left(\rho_{1}, \rho_{2}\right)=\frac{\cosh \left(\phi_{\mathbf{w}} / 2\right)}{\cosh \phi_{\mathbf{u}}} \frac{\cosh \left(\phi_{\mathbf{w}} / 2\right)}{\cosh \phi_{\mathbf{v}}} .
$$

Here, w is the Einstein sum of the two relativistically admissible velocities $\mathbf{u}$ and $\mathbf{v}$

$$
\mathbf{w}=\mathbf{u} \oplus \mathbf{v}=\frac{1}{1+\mathbf{u} \cdot \mathbf{v}}\left[\mathbf{u}+\frac{1}{\gamma_{\mathbf{u}}} \mathbf{v}+\frac{\gamma_{\mathbf{u}}}{1+\gamma_{\mathbf{u}}}(\mathbf{u} \cdot \mathbf{v}) \mathbf{u}\right]
$$

where $\gamma_{\mathbf{u}}=1 / \sqrt{1-|\mathbf{u}|^{2}}=\cosh \phi_{\mathbf{u}}$ is the Lorentz factor. The rapidity $\phi_{\mathbf{w}}$ satisfies the Cosine law of hyperbolic geometry

$\cosh \phi_{\mathbf{w}}=\cosh \phi_{\mathbf{u}} \cosh \phi_{\mathbf{v}}\left(1+\hat{\mathbf{u}} \cdot \hat{\mathbf{v}} \tanh \phi_{\mathbf{u}} \tanh \phi_{\mathbf{v}}\right)$.

The hyperbolic angles $\left\{\phi_{\mathbf{u}}, \phi_{\mathbf{v}}, \phi_{\mathbf{w}}\right\}$ form a hyperbolic triangle, which is shown in Fig,1 by Chen et al. [10]. 


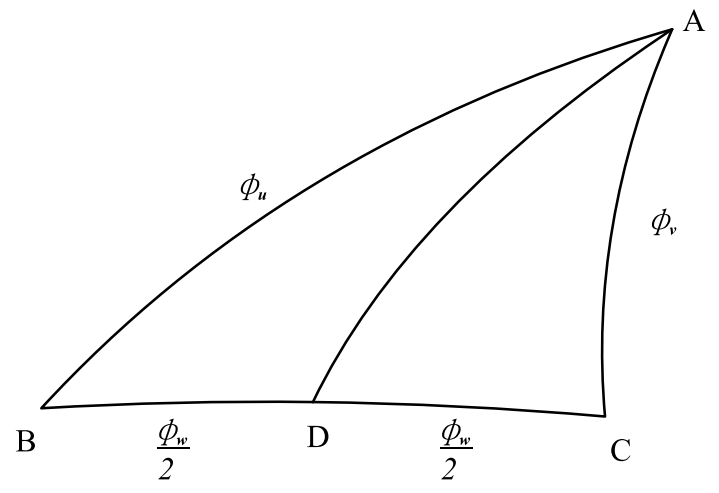

FIG. 1: The hyperbolic triangle $A B C$. Its three sides are $|A B|=\phi_{\mathbf{u}}=\tanh ^{-1}|\mathbf{u}|,|A C|=\phi_{\mathbf{v}}=\tanh ^{-1}|\mathbf{v}|$, and $|B C|=\phi_{\mathbf{w}}=\tanh ^{-1}|\mathbf{w}| . D$ is the midpoint of the side $B C$. The angle between $A B$ and $A C$ is equal to $\pi-\cos ^{-1}(\hat{\mathbf{u}} \cdot \hat{\mathbf{v}})$

\section{GEOMETRIC MEANING OF A-FIDELITY}

Theorem 1. The A-fidelity between two states $\rho_{1}$ and $\rho_{2}$ in Eq. (7) equals to

$$
\begin{aligned}
F_{A}\left(\rho_{1}, \rho_{2}\right) & =\frac{\cosh \left(\phi_{\mathbf{w}} / 2\right)}{\cosh \phi_{\mathbf{u}}} \frac{\cosh \left(\phi_{\mathbf{w}} / 2\right)}{\cosh \phi_{\mathbf{v}}} \cos ^{2} \frac{\delta}{2}, \\
& =F_{B}\left(\rho_{1}, \rho_{2}\right) \cos ^{2} \frac{\delta}{2}
\end{aligned}
$$

where $\mathbf{w}$ is defined in Eq. (9), and $\delta$ is the defect of the triangle $\triangle A B C$ in Fig 1 which satisfies [11, 12, 13]

$$
\cos \frac{\delta}{2}=\frac{1+\cosh \phi_{\mathbf{u}}+\cosh \phi_{\mathbf{v}}+\cosh \phi_{\mathbf{w}}}{4 \cosh \frac{\phi_{\mathbf{u}}}{2} \cosh \frac{\phi_{\mathbf{v}}}{2} \cosh \frac{\phi_{\mathbf{w}}}{2}} .
$$

Proof. In terms of the hyperbolic parameter, we can transform Eq. (5) into

$$
\sqrt{\rho(\mathbf{n})}=\frac{\cosh \left(\phi_{\mathbf{n}} / 2\right)}{\sqrt{2 \cosh \phi_{\mathbf{n}}}}\left[\mathbf{1}+\vec{\sigma} \cdot \hat{\mathbf{n}} \tanh \left(\phi_{\mathbf{n}} / 2\right)\right] .
$$

Then,

$$
\begin{aligned}
\sqrt{\rho_{1}} \sqrt{\rho_{2}} & =\frac{\cosh \left(\phi_{\mathbf{u}} / 2\right)}{\sqrt{2 \cosh \phi_{\mathbf{u}}}}\left[\mathbf{1}+\vec{\sigma} \cdot \hat{\mathbf{u}} \tanh \left(\phi_{\mathbf{u}} / 2\right)\right] \\
& \times \frac{\cosh \left(\phi_{\mathbf{v}} / 2\right)}{\sqrt{2 \cosh \phi_{\mathbf{v}}}}\left[\mathbf{1}+\vec{\sigma} \cdot \hat{\mathbf{v}} \tanh \left(\phi_{\mathbf{v}} / 2\right)\right] .
\end{aligned}
$$

From the relations

$$
\begin{aligned}
\operatorname{Tr}(\mathbf{1}) & =2, \\
\operatorname{Tr}(\vec{\sigma} \cdot \hat{\mathbf{n}}) & =0, \\
(\vec{\sigma} \cdot \hat{\mathbf{u}})(\vec{\sigma} \cdot \hat{\mathbf{v}}) & =\hat{\mathbf{u}} \cdot \hat{\mathbf{v}} \mathbf{1}+i \vec{\sigma} \cdot(\hat{\mathbf{u}} \times \hat{\mathbf{v}}),
\end{aligned}
$$

we have

$$
\begin{aligned}
& \operatorname{Tr}\left(\sqrt{\rho_{1}} \sqrt{\rho_{2}}\right)= \\
& \quad \frac{\cosh \frac{\phi_{\mathbf{u}}}{2} \cosh \frac{\phi_{\mathbf{v}}}{2}}{\sqrt{\cosh \phi_{\mathbf{u}} \cosh \phi_{\mathbf{v}}}}\left[1+\hat{\mathbf{u}} \cdot \hat{\mathbf{v}} \tanh \frac{\phi_{\mathbf{u}}}{2} \tanh \frac{\phi_{\mathbf{v}}}{2}\right]
\end{aligned}
$$

From the Cosine law Eq. (10), one can easily obtain

$$
\hat{\mathbf{u}} \cdot \hat{\mathbf{v}}=\frac{\cosh \phi_{\mathbf{w}}-\cosh \phi_{\mathbf{u}} \cosh \phi_{\mathbf{v}}}{\sinh \phi_{\mathbf{u}} \sinh \phi_{\mathbf{v}}} .
$$

Substituting it into Eq. (16), along with some relations between trigonometric functions of hyperbolic geometry, we derive Eq. (11), which ends the proof.

\section{UPPER BOUND OF BURES FIDELITY}

In the above section, we obtain a geometric picture of the A-fidelity in hyperbolic geometry. It is also shown that, for a qubit system, the factor connecting the Afidelity and Bures fidelity has a simple geometric meaning. Inserting Eq. (4) into Eqs. (8) and (12), one obtains

$$
\begin{aligned}
F_{B} & =\frac{1}{2}\left[1+\mathbf{u} \cdot \mathbf{v}+\sqrt{1-|\mathbf{u}|^{2}} \sqrt{1-|\mathbf{v}|^{2}}\right] \\
\cos ^{2} \frac{\delta}{2} & =\frac{\left[2 F_{B}+\sqrt{1-|\mathbf{u}|^{2}}+\sqrt{1-|\mathbf{v}|^{2}}\right]^{2}}{4\left(1+\sqrt{1-|\mathbf{u}|^{2}}\right)\left(1+\sqrt{1-|\mathbf{v}|^{2}}\right) F_{B}} .
\end{aligned}
$$

Let

$$
\begin{aligned}
f(x)= & 4[(1+\sin \alpha)(1+\sin \beta)-1] x^{2} \\
& -4(\sin \alpha+\sin \beta) x-(\sin \alpha+\sin \beta)^{2},
\end{aligned}
$$

where $\alpha=\cos ^{-1}|\mathbf{u}|$ and $\beta=\cos ^{-1}|\mathbf{v}|$. Then, we have

$$
F_{B}-\cos ^{2} \frac{\delta}{2}=\frac{1}{4(1+\sin \alpha)(1+\sin \beta) F_{B}} f\left(F_{B}\right) .
$$

Given a pair of $|\mathbf{u}|$ and $|\mathbf{v}|$, the corresponding Bures fidelity satisfies

$$
0 \leq \frac{1-\cos (\alpha+\beta)}{2} \leq F_{B} \leq \frac{1+\cos (\alpha-\beta)}{2} \leq 1 .
$$

Considering

$$
\begin{array}{r}
f(0)=-(\sin \alpha+\sin \beta)^{2} \leq 0, \\
f(1)=-(\sin \alpha-\sin \beta)^{2} \leq 0, \\
4[(1+\sin \alpha)(1+\sin \beta)-1] \geq 0,
\end{array}
$$

we have

$$
F_{B} \leq \cos ^{2} \frac{\delta}{2}
$$

Only when $\mathbf{u}=\mathbf{v}$ or $|\mathbf{u}|=|\mathbf{v}|=1$, one has $F_{B}=\cos ^{2} \frac{\delta}{2}$. This result shows that the defect of $\triangle A B C$ in Fig. 11 associates with an upper bound of the Bures fidelity.

On the other hand, from our result in Eq. (11), one can obtain

$$
F_{A}=F_{B} \cos ^{2} \frac{\delta}{2} \leq F_{B}
$$

$F_{A}\left(\rho_{1}, \rho_{2}\right)=F_{B}\left(\rho_{1}, \rho_{2}\right)$, when $\rho_{1}$ commutes with $\rho_{2}$. Then, $\mathbf{u} / / \mathbf{v}, \angle B A C=\pi$, the triangle $\triangle A B C$ becomes a line and $\delta=0$. 
From Eqs. (25) and (24), we can immediately obtain

$$
F_{B}^{2} \leq F_{A} \leq F_{B}
$$

which coincides with the result in [8].

The density matrix of a qunit ( $N$-state system) [14, 15] can be written as

$$
\rho(\mathbf{m})=\frac{1}{N}\left[\mathbf{1}_{N}+\sqrt{\frac{N(N-1)}{2}} \vec{\lambda} \cdot \mathbf{m}\right],
$$

where $\mathbf{1}_{N}$ denotes the $N \times N$ unit matrix, $\vec{\lambda}=$ $\left(\lambda_{1}, \lambda_{2}, \ldots, \lambda_{N^{2}-1}\right)$ are the generators of $S U(N)$, and $\mathbf{m}$ is the $\left(N^{2}-1\right)$-dimensional Bloch vector. It is expected that $\cos ^{2} \frac{\delta}{2}$ given in Eq. (12) is always the upper bound of the Bures fidelity for arbitrary $N$. We have tested the relation (24), for $N=3$ and 4 , by numerical computation. The results of $10^{5}$ random pairs of states show it is indeed the case.

\section{METRICS RELATED TO A-FIDELITY}

Fidelity by itself is not a metric, but there are many metrics built up from Bures fidelity to measure the distance between two quantum states. The most famous ones are known in the literature as the Bures angle, the Bures metric, and the gold metric [16], given by

$$
\begin{aligned}
& A(\rho, \sigma)=\arccos \sqrt{F_{B}(\rho, \sigma)} \\
& B(\rho, \sigma)=\sqrt{2-2 \sqrt{F_{B}(\rho, \sigma)}} \\
& C(\rho, \sigma)=\sqrt{1-F_{B}(\rho, \sigma)} .
\end{aligned}
$$

They satisfy the the following four axioms of a metric:

(M1). $d(x, y) \geq 0$ for all states $x$ and $y$;

(M2). $d(x, y)=0$ if and only if $x=y$;

(M3). $d(x, y)=d(y, x)$ for all states $x$ and $y$;

(M4). The triangle inequality: $d(x, y) \leq d(x, z)+$ $d(y, z)$ for all states $x, y$ and $z$.

For a qubit system, it is easy to prove a common ground of the above three metrics

$$
\begin{aligned}
& \lim _{\mathbf{u}, \mathbf{v} \rightarrow 0} A\left(\rho_{1}, \rho_{2}\right)=\lim _{\mathbf{u}, \mathbf{v} \rightarrow 0} B\left(\rho_{1}, \rho_{2}\right)=\lim _{\mathbf{u}, \mathbf{v} \rightarrow 0} C\left(\rho_{1}, \rho_{2}\right) \\
& =\frac{1}{2}|\mathbf{u}-\mathbf{v}|
\end{aligned}
$$

Namely, when $|\mathbf{u}|$ and $|\mathbf{v}|$ approach 0, all the above limits are the trace distance of two states of a qubit [1]. It is easy to prove

$$
\lim _{\mathbf{u}, \mathbf{v} \rightarrow 0} F_{A}\left(\rho_{1}, \rho_{2}\right)=\lim _{\mathbf{u}, \mathbf{v} \rightarrow 0} F_{B}\left(\rho_{1}, \rho_{2}\right)=1-\frac{1}{4}|\mathbf{u}-\mathbf{v}|^{2}(.30)
$$

This result suggests, if we introduce,

$$
\begin{aligned}
& \mathcal{A}(\rho, \sigma)=\arccos \sqrt{F_{A}(\rho, \sigma)} \\
& \mathcal{B}(\rho, \sigma)=\sqrt{2-2 \sqrt{F_{A}(\rho, \sigma)}} \\
& \mathcal{C}(\rho, \sigma)=\sqrt{1-F_{A}(\rho, \sigma)}
\end{aligned}
$$

their limits are also the trace distance when the two states approach the center of the Bloch sphere. Furthermore, they are three well-defined metrics of qubit and qunit states. Actually Raggio [8] has analytically proved that $\mathcal{B}(\rho, \sigma)$ is a metric. For $\mathcal{A}(\rho, \sigma)$ and $\mathcal{C}(\rho, \sigma)$, we have numerically verified the triangle inequality by using random $10^{5}$ sets of states for 2, 3 and 4-dimensional system separately. The analytic proof will be given in the subsequent investigation.

\section{CONCLUSION AND DISCUSSION}

We have proposed a geometric observation for the Afidelity between two states of a qubit in terms the hyperbolic parameters introduced in [10]. The A-fidelity is shown as the product of Bures fidelity and $\cos ^{2} \frac{\delta}{2}$. $\delta$ is nothing but the defect of the hyperbolic triangle $\triangle A B C$ plotted in Fig 1. And $\cos ^{2} \frac{\delta}{2}$ is proved as an upper bound of the Bures fidelity between two states. We also discussed the definitions of metrics based on the A-fidelity. And the numerical result sustains our expectation.

In Ref. [17], the authors have introduced an alternative fidelity for a qunit holding the same geometric observation as the qubit system. Their precept is to define the fidelity of a qunit in terms of the Bloch vectors in the formula of the qubit case. We can insert the Bloch vectors into Eq. (11), and obtain

$F_{A}\left(\rho_{1}, \rho_{2}\right)=\frac{\left[\left(1+\sqrt{1-|\mathbf{u}|^{2}}\right)\left(1+\sqrt{1-|\mathbf{v}|^{2}}\right)+\mathbf{u} \cdot \mathbf{v}\right]^{2}}{4\left(1+\sqrt{1-|\mathbf{u}|^{2}}\right)\left(1+\sqrt{1-|\mathbf{v}|^{2}}\right)}$

An alternative A-fidelity for a $N$-state system can be defined as

$$
\begin{aligned}
& \mathcal{F}_{A}(\rho, \sigma)= \\
& \frac{[(1+\sqrt{1-g(\rho, \rho)})(1+\sqrt{1-g(\sigma, \sigma)})+g(\rho, \sigma)]^{2}}{4(1+\sqrt{1-g(\rho, \rho)})(1+\sqrt{1-g(\sigma, \sigma)})},
\end{aligned}
$$

where

$$
g(\rho, \sigma)=\frac{N \operatorname{Tr}(\rho \sigma)-1}{N-1}
$$

Substituting Eq. (27) into Eq. (33), it is easy to prove that $\mathcal{F}_{A}(\rho, \sigma)$, which takes the form in Eq. (32), can also be written in the form as Eq. (11). Therefore, it holds the same geometric picture as the fidelity of the qubit system, and is a operable definition for a $N$-state system.

\section{Acknowledgments}

This work is supported by the New teacher Foundation of Ministry of Education of P.R.China (Grant No. 20070248087). J.L.C is supported in part by NSF of 
China (Grant No. 10605013), and Program for New Century Excellent Talents in University, and the Project- sponsored by SRF for ROCS, SEM. F.L.Z thank Ning Ou-Yang for her help in grammar.
[1] M. A. Nielsen and I. L. Chuang, Quantum Computation and Quantum Information (Cambridge University Press, Cambridge, 2000).

[2] V. Vedral, M. B. Plenio, M. A. Rippin, and P. L. Knight, Phys. Rev. Lett. 78, 2275 (1997).

[3] H. Barnum, C. M. Caves, C. A. Fuchs, R. Jozsa, and B. Schumacher, Phys. Rev. Lett. 76, 2818 (1996).

[4] D. Bures, Trans. Am. Math. Soc. 135, 199 (1969).

[5] A. Uhlmann, Rep. Math. Phys 9, 273 (1976).

[6] A. Uhlmann, Rep. Math. Phys. 24, 229 (1986).

[7] A. Uhlmann, Ann. Phys. (Leipzig) 501, 63 (1989).

[8] G. A.Raggio, in Lecture notes in mathematics (1984), p. 1055.

[9] S. Luo and Q. Zhang, Phys. Rev. A 69, 032106 (2004).

[10] J. L. Chen, L. Fu, A. A. Ungar, and X. G. Zhao, Phys.
Rev. A 65, 024303 (2002).

[11] J. L. Chen and M. L. Ge, J. Geom. Phys. 25, 341 (1998).

[12] P. K. Aravind, Am. J. Phys. 65, 634 (1997).

[13] A. A. Ungar, Found. Phys. 27, 881 (1997).

[14] D. Kaszlikowski, P. Gnaciński, M. Żukowski, W. Miklaszewski, and A. Zeilinger, Phys. Rev. Lett. 85, 4418 (2000).

[15] J. L. Chen, D. Kaszlikowski, L. C. Kwek, C. H. Oh, and M. Żukowski, Phys. Rev. A 64, 052109 (2001).

[16] A. Gilchrist, N. K. Langford, and M. A. Nielsen, Phys. Rev. A 71, 062310 (2005).

[17] J. L. Chen, L. Fu, A. A. Ungar, and X. G. Zhao, Phys. Rev. A 65, 054304 (2002). 